\title{
Fyn knockdown inhibits migration and invasion in cholangiocarcinoma through the activated AMPK/mTOR signaling pathway
}

\author{
SHAO-CHENG LYU, DONG-DONG HAN, XIAN-LIANG LI, JUN MA, \\ QIAO WU, HONG-MENG DONG, CHUN BAI and QIANG HE \\ Department of Hepatobiliary Surgery, Beijing Chao-Yang Hospital, Capital Medical University, Beijing 100020, P.R. China
}

Received January 13, 2016; Accepted July 3, 2017

DOI: $10.3892 / \mathrm{ol} .2017 .7542$

\begin{abstract}
Cholangiocarcinoma (CCA) is a rare and fatal tumor. In previous decades, there has been a steady increase in the incidence and mortality rates of this tumor worldwide. Metastasis is regarded as the major factor that contributes to poor prognosis in CCA patients. Studies therefore aim to develop novel therapeutic targets to control CCA metastasis. Fyn is known to enhance expression and promote metastasis in various cancers, including pancreatic cancer, prostate cancer and colorectal cancer. However, the exact function and mechanism of Fyn in CCA metastasis remains unclear. In the present study, mRNA and protein expression levels of Fyn, AMP-activated protein kinase (AMPK), phosphorylated (p-) AMPK, mammalian target of rapamycin (mTOR) and p-mTOR were measured, using the reverse transcription-quantitative polymerase chain reaction and western blot analysis, in CCA tissues and cell lines. In addition, Transwell assays were used to determine the migratory and invasive abilities of human CCA QBC939, following transfection. In the present study, it was found that Fyn was overexpressed in CCA cell lines. Fyn knockdown inhibited CCA cell migration and invasion. Furthermore, it was demonstrated that Fyn knockdown induces phosphorylation of AMPK, inhibits downstream phosphorylation of mTOR, and activate the AMPK/mTOR signaling pathway. Compound $\mathrm{C}$, an AMPK inhibitor, inhibited the AMPK/mTOR signaling pathway, and reversed the effect of Fyn knockdown on migration and invasion of CCA cells. In conclusion, the present study suggests that Fyn knockdown inhibits cell migration and invasion by regulating the
\end{abstract}

Correspondence to: Dr Qiang He, Department of Hepatobiliary Surgery, Beijing Chao-Yang Hospital, Capital Medical University, 8 Gongren Tiyuchang Nanlu Street, Chaoyang, Beijing 100020, P.R. China

E-mail: heqiang349@sina.com

Key words: Fyn, cholangiocarcinoma, migration, invasion, AMPK, mTOR
AMPK/mTOR signaling pathway in CCA cell lines and that Fyn knockdown is a potential target for anti-CCA therapy.

\section{Introduction}

Cholangiocarcinoma (CCA) is the second most common primary liver malignancy after hepatocellular carcinoma in 2012 worldwide (1). It is defined as a rare and fatal tumor, which in previous decades has demonstrated a steady increase in the incidence and mortality rate worldwide (2). The early clinical symptoms of CCA are challenging to identify, resulting in a lack of accurate diagnosis at the early stage, and inability to control the incidence rate (3). Patients with early stage CCA are usually recommended to undergo surgical resection, which is considered to be the most favorable therapy to achieve good prognosis and, to a certain extent, prolong the survival of CCA patients $(4,5)$. While the prognosis of advanced CCA patients remains poor, metastasis is regarded as the major factor that contributes to their poor outcome (5-7). Tumor metastasis is a complex and multistep biological process, wherein metastasis-promoting genes and inhibition of metastasis suppressors play critical roles during the entire metastatic process (8). A number of genes can affect the tumor metastatic process, and therefore, researchers aim to develop novel therapeutic targets to control CCA metastasis.

Fyn, a member of the Src tyrosine kinase family (SFK), is known to be involved in several biological activities associated with cancer. Fyn expression is upregulated in multiple human cancers including prostate cancer, glioblastoma, chronic myelogenous leukemia, melanoma and squamous cell carcinoma (9-13). Fyn expression is also associated with tumor metastasis. Chen et al $(14,15)$ have found that Fyn expression is upregulated in pancreatic cancer and that Fyn requires HnRNPA2B1 and Sam68 to coordinate and regulate metastasis in pancreatic cancer. In advanced prostate cancer, Fyn was found to enhance the neuroendocrine phenotype and increase visceral metastasis (16). In colorectal cancer, Fyn is induced by cellular prion protein accelerates colorectal cancer metastasis (17). In breast cancer, Fyn expression is induced by Ras/PI3K/Akt signaling and is essential for enhanced breast cancer migration and invasion (18). 
The exact function and mechanism of Fyn in CCA metastasis remains unclear. In the present study, the expression of Fyn in CCA cell lines and the association with CCA cell migration and invasion were investigated. The results showed that Fyn is upregulated in CCA cell lines and that downregulation of Fyn suppresses CCA cell migration and invasion. Mechanistic studies revealed that Fyn inhibited AMPK/mTOR signaling, which promoted CCA cell migration and invasion. The present results confirmed that Fyn blockade is a potential target for anti-CCA therapy.

\section{Materials and methods}

Cell culture. The normal bile duct HIBEC cell line and the human CCA QBC939, RBE, CCLP1 and HCCC-9810 cell lines were purchased from the Cell Bank of Chinese Academy of Sciences (Shanghai, China). Cells were cultured in RPMI-1640 medium with 10\% fetal bovine serum (FBS), penicillin $(100 \mathrm{U} / \mathrm{ml})$ and streptomycin $(100 \mu \mathrm{g} / \mathrm{ml})(\mathrm{Gibco}$; Thermo Fisher Scientific, Inc., Waltham, MA, USA) at $37^{\circ} \mathrm{C}$ in a humidified atmosphere containing $5 \% \mathrm{CO}_{2}$.

Fyn knockdown. Suppression of Fyn (NM_002037.5) expression was achieved using short hairpin RNA (shRNA) lentiviral transduction particles. The short hairpin RNA (shRNA) of Fyn (NM_002037.5; shFyn) was amplified from normal human genomic DNA using the following primers (Genewiz, Inc., Suzhou, China): shRNA forward (BamHI), 5'-GATCCCACA GGTGGCTGCAGGAATGGTCAAGAGCCATTCCTGCAG CCACCTGTGTTTTTTTG-3' and reverse, (EcoRI), 5'-AAT TCCAAAAAAACACAGGTGGCTGCAGGAATGGCTCTT GACCATTCCTGCAGCCACCTGTGG-3'. The shFyn was cloned into pLVX-shRNA1 vectors (Clontech Laboratories, Inc., Mountain View, CA, USA), termed pLVX-shFyn. Recombinant pLVX-shFyn and pHelper 1.0 and 2.0 plasmids (Clontech Laboratories, Inc.) were generated by the transient transfection of HEK 293T cells $\left(2 \times 10^{6}\right.$ cells; Cell Bank of Chinese Academy of Sciences), using Lipofectamine 2000 (Invitrogen; Thermo Fisher Scientific, Inc.) following the manufacturer's protocol, and the lentivirus was packaged in accordance with a previous study (19). For stable transfection, QBC939 cells were infected with the virus supernatant fluid along with $8 \mu \mathrm{g} / \mathrm{ml}$ polybrene and selected in $0.5 \mu \mathrm{g} / \mathrm{ml}$ puromycin for one week, followed by $0.2 \mu \mathrm{g} / \mathrm{ml}$ puromycin for one month. The stable cells were harvested 24 or $48 \mathrm{~h}$ after transfection for additional analysis.

Cell groups and treatment. The stable QBC939 cells expressing shFyn were termed shFyn. The QBC939 cells transfected with lentivirus expressing negative control shRNA were termed shNC. To validate that Fyn promotes CCA metastasis through the activated AMPK/mTOR signaling pathway, AMPK inhibitor compound C (10 $\mu \mathrm{M}$; Calbiochem, San Diego, CA, USA) was used to inhibit the AMPK/mTOR signaling and $1 \%$ dimethyl sulphoxide (DMSO) was used as negative control (NC). In the present study, 2x105 shFyn-transfected QBC939 cells were cultured at $37^{\circ} \mathrm{C}$ for $24 \mathrm{~h}$ in a humidified atmosphere containing $5 \% \mathrm{CO}_{2}$, and subsequently with $10 \mu \mathrm{l}$ compound $\mathrm{C}$ or DMSO at $37^{\circ} \mathrm{C}$ for 24 or $48 \mathrm{~h}$. Then, the shFyn-transfected QBC939 cells were harvested for additional analysis.
Reverse transcription-quantitative polymerase chain reaction (RT-qPCR) analysis. Total RNA was extracted from cultured QBC939 cells using TRIzol (Invitrogen; Thermo Fisher Scientific, Inc.) according to the manufacturer's instructions, and the RNA was reverse transcribed into cDNA at $42^{\circ} \mathrm{C}$ for 15 min using AffinityScript QPCR cDNA Synthesis kit (Agilent Technologies, Inc., Santa Clara, CA, USA). Fyn RNA expression was detected by RT-qPCR using Brilliant II SYBR Green QPCR Master Mix kit (Agilent Technologies, Inc.). Amplification was performed using the following PCR conditions: Preheating at $95^{\circ} \mathrm{C}$ for $10 \mathrm{~min}$, followed by 40 cycles of $95^{\circ} \mathrm{C}$ for $10 \mathrm{sec}, 60^{\circ} \mathrm{C}$ for $20 \mathrm{sec}$ and $72^{\circ} \mathrm{C}$ for $10 \mathrm{sec}$. Fyn and 18srRNA (internal control) primers were synthesized by Sangon Biotech (Shanghai, China). The following primer sequences were used: Fyn forward, 5'-GGG TGCTAATGTGGAGACTG-3' and reverse, 5'-GCTTTGATG CTGACTTGCAG-3'; and 18srRNA forward, 5'-CCTGGA TACCGCAGCTAGGA-3' and reverse, 5'-GCGGCGCAATAC GAATGCCCC-3'. RT-qPCR was performed on an Applied Biosystems 7500 system (Thermo Fisher Scientific, Inc.) with melt curve analysis. Gene expression was measured in triplicate. The data were analyzed using the $2^{-\Delta \Delta \mathrm{Cq}}$ method (20). Quantitative analysis was performed using the ratio of the target gene to 18srRNA, and normalized to the control.

Western blotting. Protein expression of Fyn, AMPK, phosphorylated AMPK (p-AMPK), mTOR and phosphorylated mTOR (p-mTOR) was detected by western blot analysis. The normal bile duct HIBEC cell line and human CCA QBC939, RBE, CCLP1 and HCCC-9810 cell lines were harvested for Fyn protein expression analysis. QBC939 cells, stably expressing shNC or shFyn, were harvested for Fyn, AMPK, p-AMPK, mTOR and p-mTOR analysis. All harvested cells were lysed using radioimmunoprecipitation assay buffer (Takara Biotechnology, Co., Ltd., Dalian, China), followed by protein quantification using BCA Protein Assay kit (Beyotime Institute of Biotechnology, Haimen, China). Approximately $30 \mu \mathrm{g}$ of protein sample was loaded per lane. Proteins were separated on $10 \%$ SDS-PAGE gel and transferred to a polyvinylidene difluoride membrane. The membrane was blocked in Tris-buffered saline with $1 \%$ Tween-20 buffer containing $5 \%$ skimmed dried milk and incubated at $4^{\circ} \mathrm{C}$ overnight. The primary antibodies, consisting of rabbit monoclonal anti-Fyn (dilution, 1:1,000; catalog no. ab125016), mouse monoclonal anti-AMPK (dilution, 1:500; catalog no. ab80039), rabbit monoclonal anti-p-AMPK (dilution, 1:2,000; catalog no. ab133448), rabbit polyclonal anti-mTOR (dilution, 1:2,000; catalog no. ab2732), rabbit polyclonal anti-p-mTOR (dilution, 1:1,000; catalog no. ab109268) (all from Abcam, Cambridge, MA, USA) and anti-GAPDH (dilution, 1:10,000; catalog no. KC-5G5; Kangchen Biotech Co., Ltd., Shanghai, China), were added and the mixture was incubated overnight at $4^{\circ} \mathrm{C}$ on a rocking platform. This was followed by incubation of the membrane with goat anti-mouse or goat anti-rabbit IgG $(\mathrm{H}+\mathrm{L})$ secondary antibody conjugated to horseradish peroxidase (1:5,000; catalog no. 1034-05 or 4050-05, respectively; Southern Biotech, Birmingham, AL, USA) for $2 \mathrm{~h}$. The proteins were visualized using Pierce ${ }^{\mathrm{TM}}$ ECL Western Blotting Substrate (Pierce; Thermo Fisher Scientific, Inc.) and were exposed on X-ray film. Gray scale images were then analyzed 


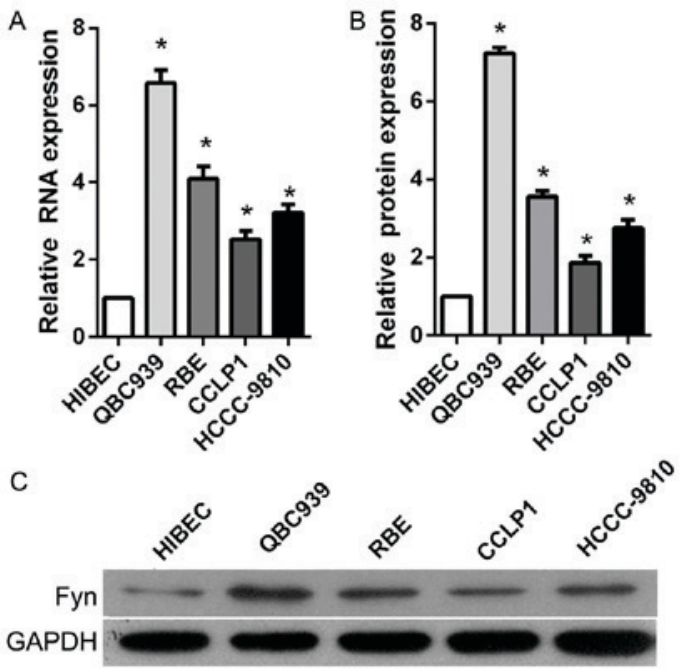

Figure 1. Fyn is overexpressed in CCA cell lines. (A) Fyn mRNA expression in the normal bile duct HIBEC cell line and in the human CCA QBC939, RBE, CCLP1 and HCCC-9810 cell lines using reverse transcription-quantitative polymerase chain reaction. GAPDH served as the internal control. (B) Fyn protein expression in the normal bile duct HIBEC cell line and in human CCA QBC939, RBE, CCLP1 and HCCC-9810 cell lines was assessed using western blot analysis. GAPDH served as internal control for western blotting. (C) Representative images of levels of Fyn protein as detected by western blot analysis. Data are represented as the mean \pm standard deviation of three independent experiments. ${ }^{*} \mathrm{P}<0.05$ vs. HIBEC. CCA, cholangiocarcinoma.

using Image J software (National Institutes of Health, Bethesda, MD, USA). The expression levels of proteins of interest were normalized to the expression of GAPDH.

Transwell migration and invasion assays. Cell migration and invasion were assessed using a Transwell migration assay. For the migration assay, the suspension $(200 \mu 1)$ containing $1 \times 10^{5}$ QBC939 cells, stably expressing shNC or shFyn, was dispensed into the upper chamber $(8 \mu \mathrm{m}$ pore size; $\mathrm{BD}$ Biosciences, San Diego, CA, USA) and RPMI-1640 medium containing $10 \%$ FBS was added to the lower chamber of the Transwell. The chamber was incubated at $37^{\circ} \mathrm{C}$ for $48 \mathrm{~h}$ in a humidified atmosphere containing $5 \% \mathrm{CO}_{2}$. Following, cells were fixed with $4 \%$ paraformaldehyde for $15 \mathrm{~min}$ at $25^{\circ} \mathrm{C}$ and stained with $0.1 \%$ crystal violet in $20 \%$ ethanol for $10 \mathrm{~min}$ at $25^{\circ} \mathrm{C}$. Images were captured using a LEICA light microscope (magnification, $\mathrm{x} 200$ ). The number of migrating cells in the center and five surrounding independent fields were counted, and average counts were calculated as the migrating cell numbers. For the invasion assay, the artificial substrate Matrigel (BD Biosciences) was layered in the Transwell chamber and $1 \times 10^{5}$ QBC939 cells from each group were dispensed into the upper chamber followed by incubation at $37^{\circ} \mathrm{C}$ for $48 \mathrm{~h}$ in a humidified atmosphere containing $5 \% \mathrm{CO}_{2}$. The subsequent treatment procedures followed those used for the migration assays. Each experiment was performed with three wells and the same experiment was measured in triplicate.

Statistical analysis. Statistical analyses were performed using SPSS version 19.0 (IBM Corp., Armonk, NY, USA). Quantitative data are presented as the mean \pm standard deviation. Fisher's least significant difference test was used to control for the multiple comparisons of comparing all CCA cell lines

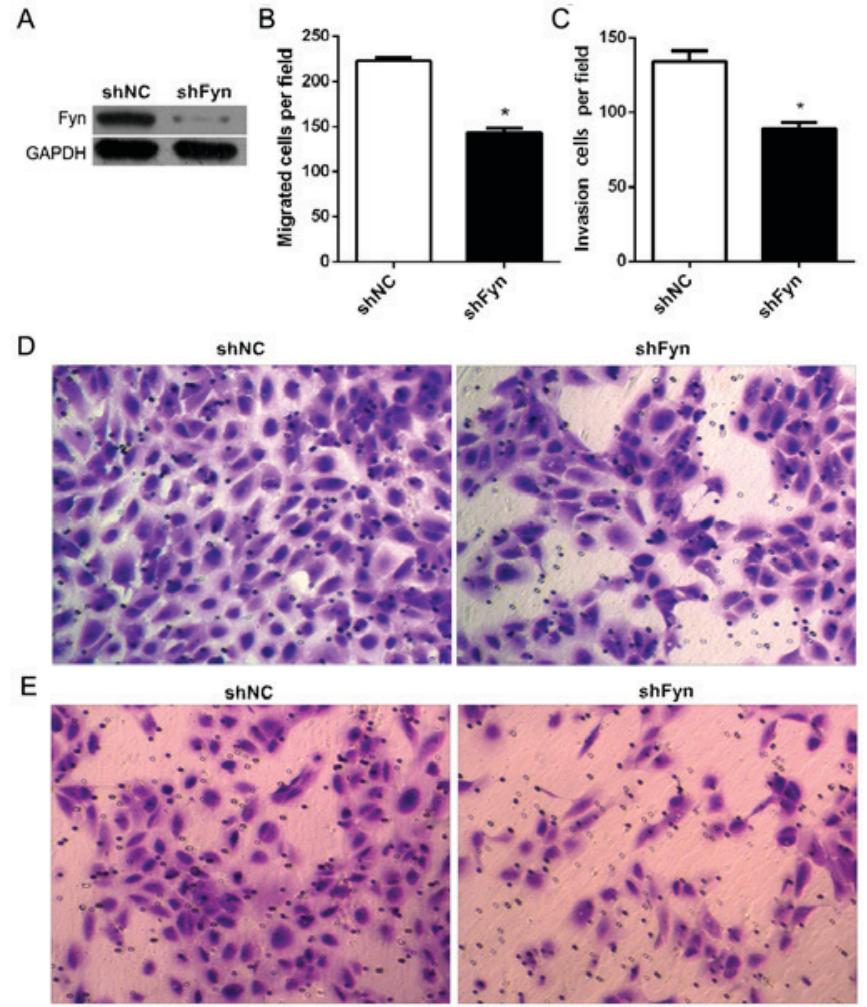

Figure 2. Fyn knockdown inhibited cholangiocarcinoma cell migration and invasion. (A) Fyn expression in QBC939 cells was examined by western blotting after transfection with shRNA for $48 \mathrm{~h}$. GAPDH served as an internal control. (B) The mean number of migrating cells per field for indicated experimental groups. (C) The mean number of invading cells per field for indicated experimental groups. (D) Migration assays of QBC939 cells Magnification, x200. (E) Invasion assays of QBC939 cells. Magnification, $\mathrm{x} 200$. Data represent the mean \pm standard deviation of three independent experiments. ${ }^{*} \mathrm{P}<0.05$ vs. shNC. shNC, control short hairpin RNA; shFyn, short hairpin RNA against Fyn.

to the same HIBEC control. The differences between shNC and shFyn groups were analyzed by the Student's t-test. $\mathrm{P}<0.05$ was considered to indicate a statistically significant difference.

\section{Results}

Fyn was upregulated in CCA tissues and cell lines. To investigate the role of Fyn in the regulation of CCA cell migration and invasion, the present study determined Fyn expression levels in the normal bile duct HIBEC cell line and in human CCA QBC939, RBE, CCLP1, and HCCC-9810 cell lines using RT-qPCR and western blot analysis. The results showed that Fyn expression was significantly decreased in CCA cell lines compared with HIBEC cells, particularly QBC939 cells (Fig. 1). Based on these results, QBC939 cells were used for subsequent experiments.

Fyn knockdown inhibited CCA cell migration and invasion. To investigate the role of Fyn in the regulation of CCA cell migration and invasion, shFyn was transfected into QBC939 cells. After $48 \mathrm{~h}$ of transfection, QBC939 cells were harvested for western blot analysis. The results showed that transfection of QBC939 cells with shFyn effectively inhibited Fyn expression (Fig. 2A). Furthermore, in order 

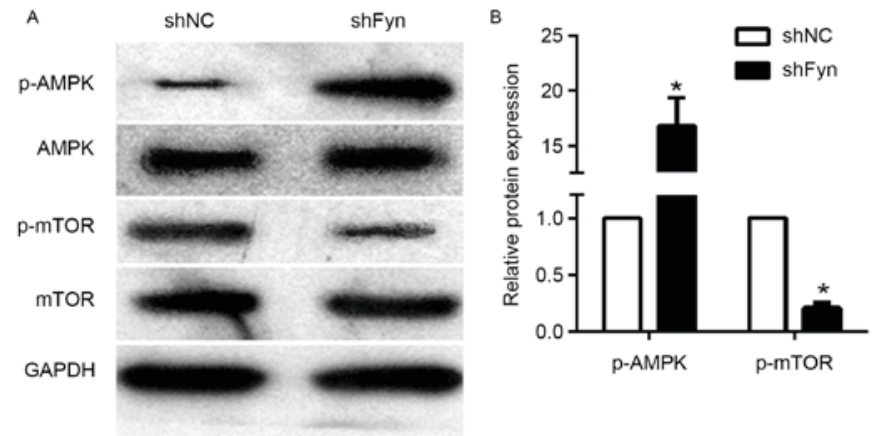

Figure 3. Fyn knockdown promoted p-AMPK expression and inhibited p-mTOR expression, suggesting that Fyn knockdown activated AMPK/mTOR signaling. (A) Expression of p-AMPK, AMPK, p-mTOR and mTOR in the cholangiocarcinoma cell line QBC939 transfected with shFyn and shNC was analyzed by western blotting. GAPDH served as an internal control. (B) Levels of p-AMPK and p-mTOR proteins in QBC939 cells transfected with shFyn and shNC. Data represent the mean \pm standard deviation for three independent experiments. " $\mathrm{P}<0.05$ vs. shNC. AMPK, AMP-activated protein kinase; mTOR, mammalian target of rapamycin; p-, phosphorylated; shNC, control short hairpin RNA; shFyn, short hairpin RNA against Fyn.
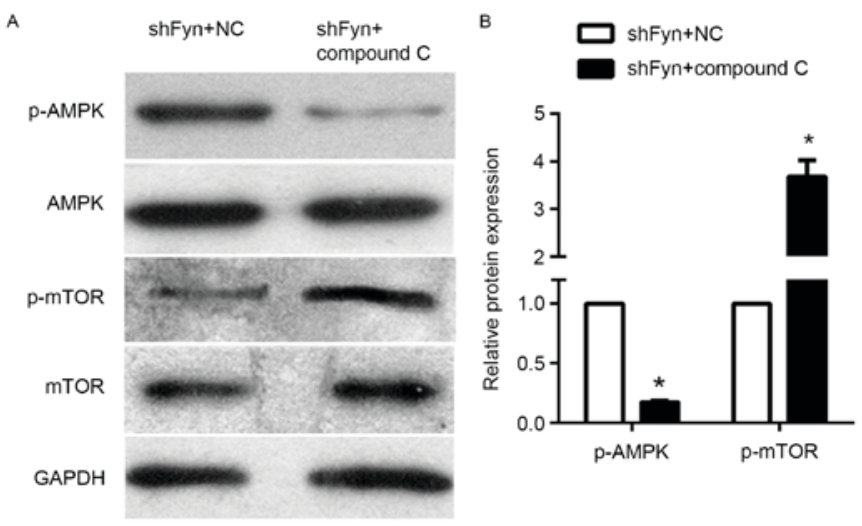

Figure 4. Compound C inhibited the AMPK/mTOR signaling pathway. (A) Expression of p-AMPK, AMPK, p-mTOR and mTOR in the cholangiocarcinoma cell line QBC939 treated with shFyn plus compound C or NC were analyzed by western blotting. GAPDH acted as an internal control. (B) Levels of phosphorylated AMPK and mTOR protein expression in QBC939 cells treated with shFyn plus compound C or NC. Data represent mean \pm standard deviation of three independent experiments. ${ }^{*} \mathrm{P}<0.05$ vs. shFyn+NC. AMPK, AMP-activated protein kinase; mTOR, mammalian target of rapamycin; p-, phosphorylated; shNC, control short hairpin RNA; shFyn, short hairpin RNA against Fyn.

to measure QBC939 cell migration and invasion, Transwell assays were used subsequent to transfection of cells with shFyn or shNC for $48 \mathrm{~h}$. The number of migrating cells after transfection with shNC or shFyn was $223 \pm 9$ and $144 \pm 13$, respectively $(\mathrm{P}<0.05$; Fig. $2 \mathrm{~B})$, and the number of invading cells was $134 \pm 17$ and $89 \pm 10$, respectively $(\mathrm{P}<0.05)$ (Fig. 2C). The Transwell migration assay showed that the ability of migration through the membrane into the lower chamber was significantly inhibited in cells transfected with shFyn compared with shNC-transfected cells ( $\mathrm{P}<0.05$; Fig. 2D). The Transwell invasion assay showed that the ability of cells to invade a Matrigel-coated membrane and migrate into the lower chamber was significantly decreased in cells transfected with shFyn compared with shNC-transfected cells $(\mathrm{P}<0.05$; Fig. 2E).
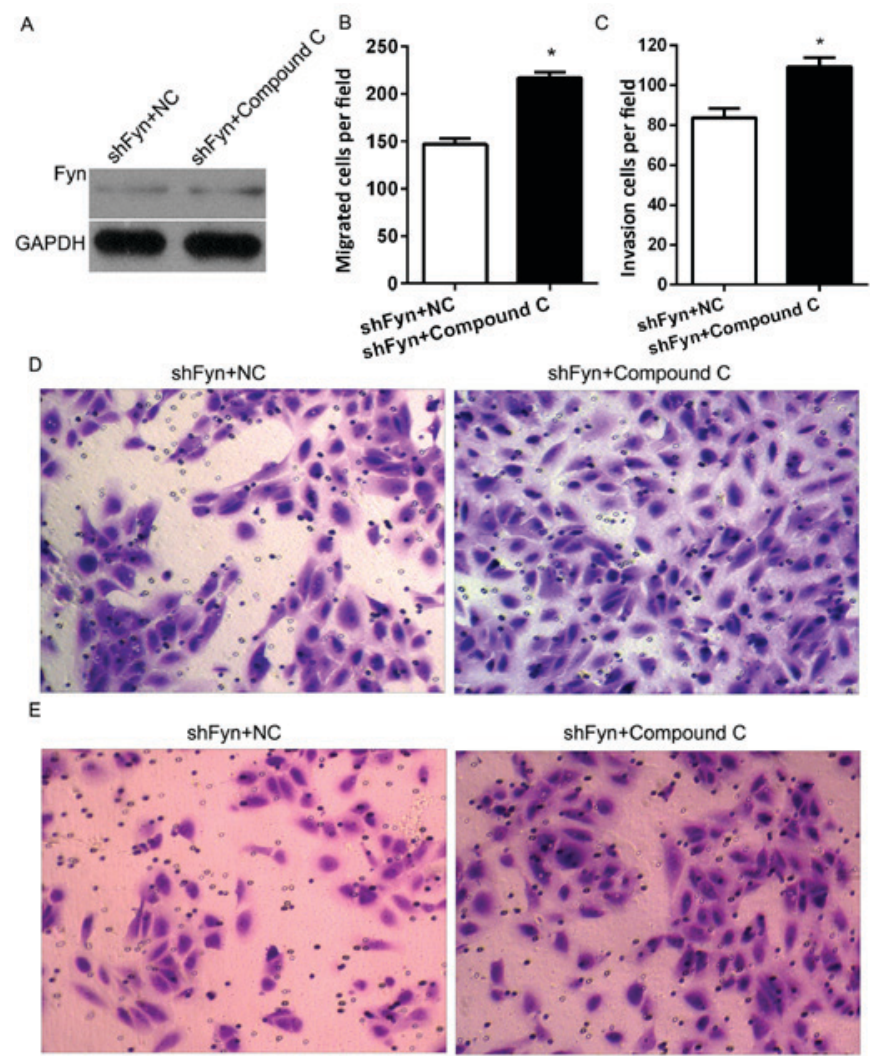

Figure 5. Compound $\mathrm{C}$ promoted cholangiocarcinoma cell migration and invasion. (A) Fyn expression in QBC939 cells transfected with shFyn was examined by western blotting after culturing with compound $\mathrm{C}$ or $\mathrm{NC}$ for $48 \mathrm{~h}$. GAPDH served as an internal control. (B) The average number of migrating cells per field for the indicated experimental groups. (C) The average number of invading cells per field for the indicated experimental groups. (D) Migration assays for QBC939 cells. Magnification, x200. (E) Invasion assays for QBC939 cells. Magnification, x200. Data represent the mean \pm standard deviation for three independent experiments. " $\mathrm{P}<0.05$ vs. shFyn+NC.

Effects of Fyn inhibition on AMPK/mTOR signaling pathway. The AMPK/mTOR signaling pathway has an important role in tumor development and metastasis. To investigate the potential mechanism of Fyn in regulation of CCA cell migration and invasion, the present study examined the effects of Fyn on AMPK/mTOR signaling. The expression of p-AMPK, AMPK, p-mTOR and mTOR in the QBC939 cells transfected with shFyn and shNC was analyzed by western blotting (Fig. 3A). The results showed that phosphorylated AMPK levels in QBC939 cells transfected with shFyn were significantly increased compared with QBC939 cells transfected with shNC. By contrast, the expression of phosphorylated mTOR was decreased, but the expression of AMPK and mTOR were not significantly different in both groups (Fig. 3B). Thus, Fyn knockdown can activate the AMPK/mTOR signaling pathway.

Compound $C$ can reverse the effect of $A M P K / m T O R$ signaling. The expression of p-AMPK, AMPK, p-mTOR and mTOR in the CCA QBC939 cell line treated with shFyn plus compound $\mathrm{C}$ or NC were analyzed by western blotting (Fig. 4A). The results of western blotting experiments showed that levels of phosphorylated AMPK in QBC939 cells treated with shFyn plus compound $\mathrm{C}$ were significantly decreased compared with QBC939 cells treated with shFyn 
plus NC. By contrast, the levels of phosphorylated mTOR were increased, whereas the expression of AMPK and mTOR were not significantly different between both the groups (Fig. 4B). Thus, compound $\mathrm{C}$ can reverse the effect the effect of Fyn shRNA on AMPK/mTOR signaling pathway in QBC939 cells.

Inhibition of AMPK/mTOR promoted CCA cell migration and invasion. In order to investigate the role of Fyn and AMPK/mTOR signaling in the regulation of CCA cell migration and invasion, the present study cultured QBC939 cells treated with shFyn plus compound C or NC. After $48 \mathrm{~h}$ of culture, Fyn expression was detected by western blotting. The results showed no differences in Fyn expression between the QBC939 cells treated with shFyn plus compound $\mathrm{C}$ and the QBC939 cells treated with shFyn plus NC (Fig. 5A). In addition, Transwell assays were used to estimate the migration and invasion of QBC939 cells treated with shFyn plus compound $\mathrm{C}$ or NC. After $48 \mathrm{~h}$ of culture, the migration numbers of QBC939 cells treated with shFyn plus compound $\mathrm{C}$ or $\mathrm{NC}$ were $147 \pm 16$ and $217 \pm 15$, respectively $(\mathrm{P}<0.05$; Fig. $5 \mathrm{~B})$, and the invasion numbers of QBC939 cells treated with shFyn plus compound $\mathrm{C}$ or $\mathrm{NC}$ were $83 \pm 11$ and $109 \pm 11$, respectively $(\mathrm{P}<0.05$; Fig. 5C). The Transwell migration assay showed that the ability of the cells to migrate through the membrane into the lower chamber was significantly inhibited in the shFyn plus compound $\mathrm{C}$ group compared to the shFyn plus NC group $(\mathrm{P}<0.05$; Fig. 5D). The Transwell invasion assay showed that the ability of the cells to pass through a Matrigel-coated membrane into the lower chamber was significantly decreased in shFyn plus compound $\mathrm{C}$ group compared to shFyn plus NC group $(\mathrm{P}<0.05$; Fig. 5E).

\section{Discussion}

CCA is a rare and fatal tumor with steadily increasing incidence and mortality rates worldwide over previous decades (1). Metastasis is regarded as the major factor that contributes to the poor prognosis of CCA patients (5). Therefore, researchers aim to develop novel therapeutic targets to control CCA metastasis. Fyn, a member of the SFK, has been found to enhance expression and promote tumor metastasis in various cancers, including pancreatic, prostate and colorectal cancers (15-17). In the present study, the function of Fyn in the regulation of CCA cell migration and invasion was investigated. The present results provide in vitro evidence that Fyn is overexpressed in CCA cell lines and that silencing Fyn inhibits CCA cell migration and invasion. These results reveal the oncogenic potential of Fyn in CCA in a manner similar to other cancers.

Little is known about the mechanisms by which Fyn induces migration and invasion of CCA cell lines. AMPK is a ubiquitous serine/threonine protein kinase that regulates tumor occurrence, development and chemoresistance through negative regulation of mTOR. Li et al (21) showed that vitamin D3 potentiates the growth inhibitory effects of metformin in human prostate DU145 cancer cells through activation of p-AMPK with subsequent inhibition of downstream mTOR signaling. Wu et al (22) demonstrated that activation of AMPK/mTOR signaling pathway is involved in autophagy-mediated cisplatin resistance in lung adenocarcinoma. AMPK/mTOR signaling also has an important role in tumor metastasis. In human non-small cell lung carcinoma (NSCLC), activated AMPK/mTOR signaling pathway suppressed the invasion and migration of NSCLC cells (23). In the present study, the effects of Fyn on AMPK/mTOR signaling were investigated. These findings demonstrate that Fyn knockdown can activate the phosphorylation of AMPK, inhibit downstream phosphorylation of mTOR, and activate the AMPK/mTOR signaling pathway.

Furthermore, the present study cultured CCA cells treated with shFyn plus compound $\mathrm{C}$ or $\mathrm{NC}$ to investigate the role of Fyn and AMPK/mTOR signaling in the regulation of CCA cell migration and invasion. As expected, the AMPK inhibitor compound $\mathrm{C}$ suppressed the AMPK phosphorylation and increased mTOR phosphorylation, without significantly affecting Fyn expression. In addition, shFyn plus compound C promoted the migration and invasion of CCA cells compared with shFyn plus NC. Thus, compound C could reverse the effect of Fyn shRNA on migration and invasion of CCA cells.

In conclusion, Fyn knockdown inhibits cell migration and invasion by activating the AMPK/mTOR signaling pathway in CCA cells. The present data only provide in vitro evidence regarding the role of Fyn in CCA cells. In vivo studies are required to further confirm the oncogenic role of Fyn and to help establish a therapeutic strategy based on Fyn targeting.

\section{References}

1. Torre LA, Bray F, Siegel RL, Ferlay J, Lortet-Tieulent J and Jemal A: Global cancer statistics, 2012. CA Cancer J Clin 65: 87-108, 2015.

2. Tyson GL, Ilyas JA, Duan Z, Green LK, Younes M, El-Serag HB and Davila JA: Secular trends in the incidence of cholangiocarcinoma in the USA and the impact of misclassification. Dig Dis Sci 59: 3103-3110, 2014.

3. Chinese Chapter of International Hepato-Pancreato-Biliary Association; Liver Surgery Group; Surgical Branch of the Chinese Medical Association; Cai JQ, Cai SW, Cong WM, Chen MS, Chen P, Chen XP, Chen YL, Chen YF, Dai CL, Huang Q, et al: Diagnosis and treatment of cholangiocarcinoma: A consensus from surgical specialists of China. J Huazhong Univ Sci Technolog Med Sci 34: 469-475, 2014.

4. Luo X, Yuan L, Wang Y, Ge R, Sun Y and Wei G: Survival outcomes and prognostic factors of surgical therapy for all potentially resectable intrahepatic cholangiocarcinoma: A large single-center cohort study. J Gastrointest Surg 18: 562-572, 2014.

5. Wang Y, Yang H, Shen C and Luo J: Cholangiocarcinoma: Prognostic factors after surgical resection in China. Int J Clin Exp Med 8: 5506-5512, 2015.

6. Yubin L, Chihua F, Zhixiang J, Jinrui O, Zixian L, Jianghua Z, Ye L, Haosheng $J$ and Chaomin L: Surgical management and prognostic factors of hilar cholangiocarcinoma: Experience with 115 cases in China. Ann Surg Oncol 15: 2113-2119, 2008.

7. Zheng-Rong L, Hai-Bo Y, Xin C, Chuan-Xin W, Zuo-Jin L, Bing T, Jian-Ping $\mathrm{G}$ and Sheng-Wei L: Resection and drainage of hilar cholangiocarcinoma: An 11-year experience of a single center in mainland China. Am Surg 77: 627-633, 2011.

8. Valastyan S and Weinberg RA: Tumor metastasis: Molecular insights and evolving paradigms. Cell 147: 275-292, 2011.

9. Posadas EM, Al-Ahmadie H, Robinson VL, Jagadeeswaran R, Otto K, Kasza KE, Tretiakov M, Siddiqui J, Pienta KJ, Stadler WM, et al: FYN is overexpressed in human prostate cancer. BJU Int 103: 171-177, 2009.

10. Lu KV, Zhu S, Cvrljevic A, Huang TT, Sarkaria S, Ahkavan D, Dang J, Dinca EB, Plaisier SB, Oderberg I, et al: Fyn and SRC are effectors of oncogenic epidermal growth factor receptor signaling in glioblastoma patients. Cancer Res 69: 6889-6898, 2009.

11. Ban K, Gao Y, Amin HM, Howard A, Miller C, Lin Q, Leng X, Munsell M, Bar-Eli M, Arlinghaus RB and Chandra J: BCR-ABL1 mediates up-regulation of Fyn in chronic myelogenous leukemia. Blood 111: 2904-2908, 2008. 
12. Huang J, Asawa T, Takato T and Sakai R: Cooperative roles of Fyn and cortactin in cell migration of metastatic murine melanoma. J Biol Chem 278: 48367-48376, 2003.

13. Kim JE, Roh E, Lee MH, Yu DH, Kim DJ, Lim TG, Jung SK, Peng $\mathrm{C}$, Cho YY, Dickinson S, et al: Fyn is a redox sensor involved in solar ultraviolet light-induced signal transduction in skin carcinogenesis. Oncogene 35: 4091-4101, 2016.

14. Chen ZY, Cai L, Bie P, Wang SG, Jiang Y, Dong JH and Li XW: Roles of Fyn in pancreatic cancer metastasis. J Gastroenterol Hepatol 25: 293-301, 2010.

15. Chen ZY, Cai L, Zhu J, Chen M, Chen J, Li ZH, Liu XD, Wang SG, Bie P, Jiang P, et al: Fyn requires HnRNPA2B1 and Sam68 to synergistically regulate apoptosis in pancreatic cancer. Carcinogenesis 32: 1419-1426, 2011

16. Gururajan M, Cavassani KA, Sievert M, Duan P, Lichterman J, Huang JM, Smith B, You S, Nandana S, Chu GC, et al: SRC family kinase FYN promotes the neuroendocrine phenotype and visceral metastasis in advanced prostate cancer. Oncotarget 6 : 44072-44083, 2015.

17. Wang Q, Qian J, Wang F and Ma Z: Cellular prion protein accelerates colorectal cancer metastasis via the Fyn-SP1-SATB1 axis. Oncol Rep 28: 2029-2034, 2012.

18. Yadav V and Denning MF: Fyn is induced by Ras/PI3K/Akt signaling and is required for enhanced invasion/migration. Mol Carcinog 50: 346-352, 2011.
19. Song JL, Zheng W, Chen W, Qian Y, Ouyang YM and Fan CY: Lentivirus-mediated microRNA-124 gene-modified bone marrow mesenchymal stem cell transplantation promotes the repair of spinal cord injury in rats. Exp Mol Med 49: e332, 2017.

20. Livak KJ and Schmittgen TD: Analysis of relative gene expression data using real-time quantitative PCR and the 2(-Delta Delta C(T)) method. Methods 25: 402-408, 2001.

21. Li HX, Gao JM, Liang JQ, Xi JM, Fu M and Wu YJ: Vitamin D3 potentiates the growth inhibitory effects of metformin in DU145 human prostate cancer cells mediated by AMPK/mTOR signalling pathway. Clin Exp Pharmacol Physiol 42: 711-717, 2015.

22. Wu T, Wang MC, Jing L, Liu ZY, Guo H, Liu Y, Bai YY, Cheng YZ, Nan KJ and Liang X: Autophagy facilitates lung adenocarcinoma resistance to cisplatin treatment by activation of AMPK/mTOR signaling pathway. Drug Des Devel Ther 9: 6421-6431, 2015.

23. Kang JI, Hong JY, Lee HJ, Bae SY, Jung C, Park HJ and Lee SK: Anti-tumor activity of yuanhuacine by regulating AMPK/mTOR signaling pathway and actin cytoskeleton organization in non-small cell lung cancer cells. PLoS One 10: e0144368, 2015.

This work is licensed under a Creative Commons Attribution-NonCommercial-NoDerivatives 4.0 International (CC BY-NC-ND 4.0) License. 